\title{
ASPECTOS MORFOLÓGICOS E FISIOLÓGICOS NO CICLISMO DE ESTRADA E MOUNTAIN BIKE CROSS-COUNTRY
}

\section{Morphological and physiological aspects in road cycling and mountain bike cross-country}

\author{
Vitor Pereira Costa ${ }^{1}$; Fernando Roberto de Oliveira ${ }^{2}$ \\ ${ }^{1}$ Centro de Educação Superior da Região Sul. Universidade do Estado de Santa Catarina - Laguna - SC - Brasil. \\ ${ }^{2}$ Núcleo de Estudos do Movimento Humano. Universidade Federal de Lavras - Lavras - MG - Brasil
}

\begin{abstract}
Resumo: As competições de ciclismo podem ser divididas basicamente em dois formatos: contra-relógio e estrada. Já o Mountain Bike (MTB) é caracterizado por ser disputado em diferentes tipos de trilhas e estradas de terra. Na tentativa de sintetizar os recentes estudos científicos no ciclismo e MTB, foi descrita esta breve revisão sobre aspectos morfofisiológicos de atletas de ambas as modalidades. Percebe-se que os ciclistas especialistas em etapas planas são altos e fortes (variação de 180 a $185 \mathrm{~cm}$ de altura, 70 a $75 \mathrm{~kg}$ de massa corporal), sendo estatisticamente significativa $(p<0,05)$ a diferença dos especialistas em montanhas $(175-180 \mathrm{~cm}, 60-66 \mathrm{~kg})$. Especula-se que as características morfológicas dos especialistas em montanhas são semelhantes aos mountain bikers ( $178 \pm 0,1 \mathrm{~cm}$ de altura, $65,3 \pm 6,5 \mathrm{~kg}$ de massa corporal, média e desvio padrão). Alguns estudos verificaram que os ciclistas atingiram a potência aeróbia máxima $\left(\mathrm{W}_{\text {máx }}\right)$ significativamente mais alta que os mountain bikers em valores absolutos e relativos à massa corporal $(p<0,05)$. Em contraste, outros demonstraram que os atletas de MTB apresentam a $W_{\text {máx }}$ e consumo de oxigênio de pico $\left(\mathrm{VO}_{\text {pico }}\right)$ relativo à massa corporal significativamente mais alto que os ciclistas $(P=0,03)$. Assim, o conflito relatado na literatura sobre as características morfofisiológicas de ciclistas e mountain bikers, se expande além das diferenças metodológicas encontradas nos estudos, sendo que talvez estejam presentes no nível competitivo e de treinamento dos diferentes atletas investigados. Dessa forma, entendemos que a interpretação das informações apresentadas serve como referências para os treinadores realizarem a prescrição do treinamento de ciclistas e mountain bikers.
\end{abstract}

Palavras-chave: Ciclismo, Mountain bike, Competições.

Abstract: Briefly, the cycling races can be divided into two events: road cycling and time trial. And Mountain Biking (MTB) is characterized by being disputed in different types of tracks and off roads terrain. In the attempt to syntheses the recent scientific studies in cycling and MTB we have described this brief review on morpho-physiological aspects between road cyclists and mountain bikers. It is clear to see that flat terrain specialists are high and strong (height range from 180 to $185 \mathrm{~cm}$, body mass $70-75 \mathrm{~kg}$ ), with significant difference $(p<0.05)$ to uphill specialists $(175-180 \mathrm{~cm}, 60-66 \mathrm{~kg})$. It is supposed that morphological features of uphill specialists are similar to mountain bikers (height $178 \mathrm{~cm}$, body mass $65.3 \pm 6.5 \mathrm{~kg}$ average and standard deviation). Some studies have verified that cyclists reached maximal power output $\left(\mathrm{W}_{\text {max }}\right)$ significantly higher than mountain bikers in absolute and relative values to body mass $(p<0.05)$. By contrast, others have showed that mountain bikers have $W_{\max }$ and peak oxygen uptake $\left(\mathrm{VO}_{\text {peak }}\right)$ relative to body mass significantly higher than cyclists $(P=0.03)$. Therefore the conflict about the morpho-physiological characteristics of cyclists and mountain bikers are expanding besides methodological differences found in the studies, and perhaps are present on competitive level and training quality. In this regard, we understand that the interpretations of the information showed are able to use as references for coaches making training programs for cyclists and mountain bikers.

Key words: Cycling, Mountain Bike, Races.

Aceito em 10/08/2009 - Rev. Educ. Fís. 2009 Jun: 145: 11-20. Rio de Janeiro - RJ - Brasil

\section{INTRODUÇÃO}

O ciclismo é uma modalidade esportiva praticada mundialmente há mais de 100 anos desde diferentes situações como o simples lazer ao complexo alto rendimento desportivo. A primeira competição oficial foi realizada em Paris, na França em 1868, sendo vencida pelo inglês James Moore(1). Desde os primeiros Jogos Olímpicos realizados em 1896, o ciclismo faz parte do programa oficial. Atualmente a União Ciclística Internacional ( $\mathrm{UCl}$ ) é a instituição responsável pela organização de 
competições mundiais e na classificação do ranking mundial dos atletas internacionais. Com a evolução do esporte, diversas modalidades derivadas do ciclismo foram criadas e a UCl durante o ano de 2008 classifica o ciclismo quanto a diferentes tipos de provas: 1) Ciclismo de estrada; 2) Ciclismo em pista; 3) Mountain bike (MTB); 4) Ciclo-cross; 5) Bmx; 6) Trials 7) Ciclismo indoor 8) Para-ciclismo (praticantes com limitações físicas). Devido aos tipos de prova, os atletas participantes realizam diferentes tipos de treinamento o que promove adaptações orgânicas específicas. Durante o treinamento e/ou competições, percebe-se que alguns atletas participam de mais de uma modalidade esportiva, sendo muitas vezes observado ciclistas de estrada praticando o MTB na modalidade Cross-country Olímpico (XCO) e/ou Ciclo-cross e vice-versa. Assim, iremos focalizar nossa discussão apenas em atletas e competições de ciclismo de estrada e no XCO.

As competições internacionais de ciclismo de estrada podem ser divididas em provas realizadas em etapas únicas ou competições que se estendem de quatro dias a três semanas consecutivas, como - Tour de France, Giro di Italia e Vuelta a Espana (2). Resumidamente, as provas podem ser divididas em dois formatos: contra-relógio e estrada. As provas de contra-relógio individual nas "grandes voltas internacionais" são realizadas em percursos com distâncias curtas, como por exemplo, o prólogo (5-10 $\mathrm{km})$; distância moderada (20-40 km) e percursos mais extensos (40-60 km). As competições de ciclismo de estrada são realizadas em um percurso cuja distância varia entre 150-230 km, também são classificadas de acordo com a dificuldade do trajeto, sendo predominantemente etapas planas, com semimontanhas e de montanhas ${ }^{(2)}$.

Diferente do ciclismo, as competições de XCO são realizadas em trilhas estreitas e sinuosas denominadas single tracks ou em estradas de terra abertas, geralmente com a presença de erosões, pedras, cascalhos, troncos, árvores e travessia em trechos com lama ${ }^{(3)}$. As primeiras competições de MTB foram disputadas na Califórnia nos EUA no início dos anos 80 sendo oficializada pela $\mathrm{UCl}$ apenas em 1990. Com o crescente número de praticantes o XCO foi acrescentado ao programa oficial dos Jogos Olímpicos em Atlanta, 1996 ${ }^{(1)}$. As competições de XCO são realizadas em circuito e consistem em um número definido de voltas, onde todos os participantes partem juntos em um único pelotão. A duração do evento está, em geral, entre 2 a $3 \mathrm{~h}$ para homens e $1 \mathrm{~h}$ e 45 min a $2 \mathrm{~h}$ e 25 min para as mulheres ${ }^{(4)}$.

Os mecanismos responsáveis pelo desempenho de atletas são tópicos de grande interesse por profissionais que trabalham com o treinamento desportivo. Como existe grande diversidade nas características naturais das provas no ciclismo de estrada e no XCO, é de interesse a discussão de fatores fisiológicos para estas modalidades. $\mathrm{Na}$ tentativa de sintetizar informações recémpublicadas nesta área, esta breve revisão abordará as características morfológicas e fisiológicas dos atletas; e a demanda fisiológica durante as competições de ciclismo de estrada e XCO.

\section{CARACTERÍSTICAS MORFOLÓGICAS E FISIOLÓGICAS DE CICLISTAS E MOUNTAIN BIKERS}

\section{Características Morfológicas}

Em breve revisão Swain ${ }^{(5)}$ apresentou as influências da massa corporal no desempenho aeróbio durante o ciclismo. O ciclismo é uma modalidade em que o custo de energia é dado por duas forças principais: a resistência do ar em terrenos planos e a gravidade em terrenos de montanha. Além disso, os ciclistas de maior estatura apresentam a resistência pela área frontal ligeiramente maior que ciclistas menores, no entanto, quando a área frontal é relativa à massa corporal, os ciclistas pequenos apresentam maior resistência do ar no plano e, conseqüentemente, estão em desvantagem ${ }^{(5)}$. Nas subidas, essa perda 
nas etapas planas é compensada devido à falta de resistência do ar, pois quanto maior a inclinação da montanha menor a velocidade. Neste momento, a força da gravidade impõe grande influência no custo de energia do atleta e os ciclistas mais leves podem se beneficiar. Para aperfeiçoar os efeitos, Swain ${ }^{(5)}$ sugere que ao relacionar as variáveis de desempenho com a massa corporal, utilizar o expoente de massa de 0,32 para o plano e 0,79 para as subidas. Assim, um ciclista profissional de $70 \mathrm{~kg}$ que atinge 430 watts de potência aeróbia máxima $\left(W_{\text {máx }}\right)$, apresenta a potência relativa à massa corporal de $6,1 \mathrm{~W} . \mathrm{kg}^{-1}$.

A massa corporal associada ao peso da bicicleta é um fator que afeta o desempenho nas subidas do ciclismo de estrada e no XCO; e a resistência do ar é reduzida em função da baixa velocidade desenvolvida neste tipo de terreno. Entretanto, Berry, Koves e Benedetto(6) $^{(6)}$ estudaram a influência da massa de diferentes bicicletas de MTB durante uma simulação no laboratório e não encontraram diferenças significativas no consumo de oxigênio $\left(\mathrm{VO}_{2}\right)$, na freqüência cardíaca (FC) e na percepção subjetiva de esforço em cargas sub-máximas. É importante lembrar que o desenho experimental tem limitações de validade ecológica, pois este estudo foi realizado na esteira, com bicicleta de suspensão traseira e dianteira que pesava $11,6 \mathrm{~kg}$ e teve aumento de 1 kg para cada teste.

A massa corporal, estatura, a superfície da área corporal e a área frontal são variáveis que podem ser determinantes para os diferentes tipos de terrenos e etapas presentes nas competições de ciclismo(5). Padilla et al. ${ }^{(7)}$ apresentaram as características fisiológicas e de desempenho em ciclistas de estrada profissionais em relação à sua constituição morfológica. Estes autores classificaram os ciclistas em quatro tipos de especialistas: atletas que se destacam nas subidas, no plano, no contra-relógio e em todos os tipos de terreno. Por outro lado, no XCO não é possível diferenciar morfologicamente os atletas em diferentes especialidades devido aos vários tipos de terreno. A superfície de área corporal e área frontal influenciam em menor proporção no XCO, pois os atletas atingem baixos valores na velocidade média ao final das provas. Costa( ${ }^{(8)}$ revela que em uma etapa da Copa do Mundo e do Campeonato Brasileiro de XCO em 2005, o primeiro colocado da categoria elite alcançou velocidades médias de $13,4 \mathrm{~km} \cdot \mathrm{h}^{-1}$ e $13,6 \mathrm{~km} \cdot \mathrm{h}^{-1}$, respectivamente. Neste sentido, os fatores envolvidos na aerodinâmica estão diretamente relacionados com o custo de energia durante a prática do ciclismo, com influência discreta em relação ao $\mathrm{XCO}^{(5,6)}$.

Portanto, o desempenho nas competições de ciclismo de estrada e XCO são determinados, em parte, pelas características morfológicas individuais. Percebe-se que os ciclistas profissionais especialistas em etapas planas são altos e fortes (variações de $180-185 \mathrm{~cm}$ e de $70-75 \mathrm{~kg}$ ), sendo estatisticamente significativa a diferença dos ciclistas especialistas em montanhas $(175-180 \mathrm{~cm}, 60-66 \mathrm{~kg})^{(2,9)}$. Assim, especula-se que as características morfológicas dos ciclistas especialistas em montanhas são semelhantes aos mountain bikers que competem no XCO (178 \pm $0,1 \mathrm{~cm}, 65,3 \pm 6,5 \mathrm{~kg})^{(10)}$. Esta similaridade pode ser observada nas competições pelo desempenho de atletas como Michael Rasmussen e Cadel Evans, ambos foram campeões mundiais no XCO e nos últimos anos ocuparam as primeiras colocações em etapas de montanha e também no ranking final das grandes voltas do ciclismo mundial(11).

\section{Variáveis Máximas de Aptidão Aeróbia}

Lucia, Hoyos e Chicharro(9) apresentam que os valores de $\mathrm{W}_{\text {máx }}$ e $\mathrm{VO}_{2 \text { máx }}$ são bastante expressivos para ciclistas profissionais, sendo protocolodependente: estágios com duração curta, os valores ficam próximos a 450 - $500 \mathrm{~W}\left(6,5-7,5 \mathrm{~W} \cdot \mathrm{kg}^{-1}\right)$ e estágios mais longos, 400 - $450 \mathrm{~W}(6,0$ - 6,5 W. $\left.\mathrm{kg}^{-1}\right)$. A TABELA 1 apresenta as variáveis aeróbias máximas identificadas em ciclistas profissionais que normalmente disputam as principais voltas do ciclismo mundial. 
TABELA 1

VARIÁVEIS MÁXIMAS E SUB-MÁXIMAS DE CICLISTAS PROFISSIONAIS SEGUNDO DIFERENTES AUTORES

\begin{tabular}{|c|c|c|c|c|c|c|c|}
\hline & $\begin{array}{c}\text { Wilber et } \\
\text { al. }{ }^{(16)}\end{array}$ & $\begin{array}{l}\text { Lucia et } \\
\text { al. }\end{array}$ & $\begin{array}{c}\text { Padilla et } \\
\text { al. }^{(7)}\end{array}$ & $\begin{array}{c}\text { Padilla et } \\
\text { al. }{ }^{(29)}\end{array}$ & $\begin{array}{c}\text { Padilla et } \\
\text { al. }^{\left({ }^{30)}\right.}\end{array}$ & Lee et al. ${ }^{(10)}$ & $\begin{array}{c}\text { Lucia et } \\
\text { al. }^{(28)}\end{array}$ \\
\hline Sujeitos (n) & 10 & 25 & 24 & 18 & 17 & 7 & 8 \\
\hline $\mathrm{VO}_{2 \text { máx }}\left(\mathrm{I} \cdot \mathrm{min}^{-1}\right)$ & $5,1 \pm 0,4$ & $5,1 \pm 0,6$ & $5,6 \pm 0,3$ & $\mathrm{NI}$ & $\mathrm{NI}$ & $5,4 \pm 0,1$ & $5,1 \pm 0,4$ \\
\hline $\mathrm{VO}_{2 \text { máx.kg }}{ }^{1}\left(\mathrm{ml} \cdot \mathrm{kg} \cdot^{-1} \cdot \mathrm{min}^{-1}\right)$ & $70,3 \pm 3,2$ & $73,9 \pm 7,4$ & $74,4 \pm 3,0$ & $\mathrm{NI}$ & $\mathrm{NI}$ & $73,0 \pm 3,4$ & $74,0 \pm 5,8$ \\
\hline$W_{\text {máx }}(w)$ & $470 \pm 35$ & $466 \pm 31$ & $432 \pm 43$ & $439 \pm 45$ & $433 \pm 48$ & $431 \pm 12$ & $501 \pm 25$ \\
\hline$W_{\text {máx.kg }}{ }^{-1}\left(\mathbf{w} \cdot \mathbf{k g}^{-1}\right)$ & $6,5 \pm 0,3$ & $6,7 \pm 0,4$ & $6,3 \pm 0,3$ & $6,4 \pm 0,3$ & $6,3 \pm 0,3$ & $5,8 \pm 0,3$ & $\mathrm{NI}$ \\
\hline$W^{\prime} L_{1}(w)$ & $\mathrm{NI}$ & $262 \pm 36$ & $\mathrm{NI}$ & $334 \pm 50$ & $332 \pm 52$ & $\mathrm{NI}$ & $\mathrm{NI}$ \\
\hline$W^{\prime} L_{2}(w)$ & $321 \pm 17$ & $386 \pm 25$ & $\mathrm{NI}$ & $386 \pm 52$ & $378 \pm 55$ & $348 \pm 16$ & $\mathrm{NI}$ \\
\hline
\end{tabular}

Valores expressos em média e desvio padrão. $\mathbf{V O}_{2 \text { máx }}$ (consumo máximo de oxigênio), $\mathbf{V O}_{2 \text { máx. }} \mathbf{k g}^{-1}$ (consumo máximo de oxigênio relativo), $\mathbf{W}_{\text {máx }}$ (potência aeróbia máxima), $\mathbf{W}_{\text {máx. }} \mathbf{k g}^{-1}$ (potência aeróbia máxima relativa), $\mathbf{W L L}_{1}$ (potência no primeiro limiar de lactato), $\mathbf{W L L}_{2}$ (potência no segundo limiar de lactato.

TABELA 2

VARIÁVEIS MÁXIMAS E SUB-MÁXIMAS DE CICLISTAS ESPECIALISTAS

\begin{tabular}{|c|c|c|c|c|}
\hline & Plano & Contra-relógio & Todos os terrenos & Montanhas \\
\hline Sujeitos (n) & 5 & 4 & 6 & 9 \\
\hline $\mathrm{VO}_{2 \operatorname{máx}}\left(\mathrm{I} \cdot \mathrm{min}^{-1}\right)$ & $5,7 \pm 0,4^{a}$ & $5,7 \pm 0,5^{a}$ & $5,4 \pm 0,3$ & $5,1 \pm 0,4$ \\
\hline $\mathrm{VO}_{2 \text { máx.kg }}{ }^{1}\left(\mathrm{ml} \cdot \mathbf{k g}-^{-1} \cdot \mathrm{min}^{-1}\right)$ & $74,4 \pm 3,0$ & $79,2 \pm 1,1^{b}$ & $78,9 \pm 1,9^{b}$ & $80,9 \pm 3,9^{b}$ \\
\hline $\mathbf{W}_{\text {máx }}(\mathbf{w})$ & $461 \pm 39^{c}$ & $457 \pm 46^{c}$ & $432 \pm 27$ & $404 \pm 34$ \\
\hline $\mathbf{W}_{\text {máx.kg }}{ }^{-1}\left(\mathbf{w} \cdot \mathrm{kg}^{-1}\right)$ & $6,0 \pm 0,3^{d}$ & $6,4 \pm 0,1$ & $6,4 \pm 0,2$ & $6,5 \pm 0,3$ \\
\hline $\mathrm{WLL}_{1}(\mathrm{w})$ & $356 \pm 31$ & $357 \pm 41$ & $322 \pm 43$ & $308 \pm 46$ \\
\hline $\mathrm{WLL}_{2}(w)$ & $417 \pm 45^{\mathrm{e}}$ & $409 \pm 46^{f}$ & $366 \pm 38$ & $356 \pm 41$ \\
\hline
\end{tabular}

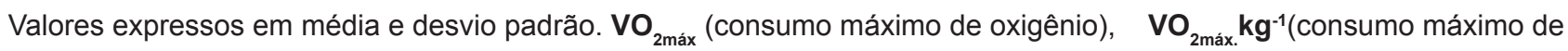
oxigênio relativo), $\mathbf{W}_{\text {máx }}$ (potência aeróbia máxima), $\mathbf{W}_{\text {máx. }} \mathbf{k g}^{-1}$ (potência aeróbia máxima relativa), $\mathbf{W L L}_{\mathbf{1}}$ (potência no primeiro limiar de lactato), $\mathbf{W L L}_{2}$ (potência no segundo limiar de lactato.(Padilla et al. (7). a, c, d, e, f Diferença significativa dos especialistas em montanhas, ${ }^{b}$ diferença significativa dos especialistas no plano, ambos $(p<0,05)$.

Ao serem classificados pelo tipo de especialidade mencionado anteriormente no estudo de Padilla, Mujika, Cuesta e Goiriena(7), percebem-se as diferenças nos valores de algumas variáveis fisiológicas entre os ciclistas $(p<0,05)$ (TABELA 2).

$\mathrm{Na}$ TABELA3 percebe-se que alguns estudos sobre atletas de XCO discordam dos valores da $\mathrm{W}_{\text {max }}$ atingida pelos ciclistas apresentados anteriormente ${ }^{(12,13)}$. Impellizzeri et al. ${ }^{(14)}$, Stapelfeldt et al. ${ }^{(15)}$ e Baron ${ }^{(4)}$ utilizaram protocolos semelhantes com estágios extensos e incrementos de carga maiores, no entanto, observa-se que os valores de $\mathrm{W}_{\text {máx }}$ são inferiores aos ciclistas na TABELA 1. Ao comparar as características fisiológicas de ciclistas e mountain bikers, Wilber et al. ${ }^{(16)}$ verificaram que os ciclistas atingiram a $\mathrm{W}_{\text {máx }}$ significativamente mais alta que os mountain bikers em valores absolutos e relativos à massa corporal $(p$ $<0,05)$. Em contraste, Lee et al. ${ }^{(10)}$ demonstraram que 
TABELA 3

VARIÁVEIS AERÓBIAS MÁXIMAS E SUB-MÁXIMAS IDENTIFICADAS EM MOUNTAIN BIKERS

\begin{tabular}{|c|c|c|c|c|c|c|c|c|}
\hline & $\begin{array}{c}\text { Heller e } \\
\text { Novotny }^{(27)}\end{array}$ & $\begin{array}{l}\text { Wilber } \\
\text { et al. }{ }^{(16)}\end{array}$ & Baron ${ }^{(4)}$ & $\begin{array}{l}\text { Lee } \\
\text { al. }^{\left({ }^{(10)}\right.}\end{array}$ & $\begin{array}{c}\text { Impellizzeri } \\
\text { et al. }{ }^{(14)}\end{array}$ & $\begin{array}{c}\text { Stapelfeldt } \\
\text { et al. }{ }^{(15)}\end{array}$ & $\begin{array}{c}\text { Impellizzeri } \\
\text { et al. }{ }^{(12)}\end{array}$ & $\begin{array}{c}\text { Impellizzeri } \\
\text { et al. }{ }^{(13)}\end{array}$ \\
\hline $\begin{array}{l}\text { Sujeitos } \\
\text { (n) }\end{array}$ & 10 & 10 & 25 & 7 & 5 & 9 & 13 & 15 \\
\hline $\begin{array}{l}\mathrm{VO}_{2 \text { max }} \\
\left(\text { I.min }^{-1}\right)\end{array}$ & $4,9 \pm 0,3$ & $5,0 \pm 0,4$ & 4,74 & $5,1 \pm 0,5$ & $4,9 \pm 0,4$ & 4,61 & $4,6 \pm 0,5$ & $5,1 \pm 0,5$ \\
\hline $\begin{array}{c}\mathrm{VO}_{2 \text { max..kg }}{ }^{1} \\
\left(\mathrm{ml.kg.-1}^{-1} \text {. }\right. \\
\left.\mathrm{min}^{-1}\right)\end{array}$ & $74,1 \pm 3,4$ & $70,0 \pm 3,7$ & $68,4 \pm 3,8$ & $78,3 \pm 4,4$ & $75,2 \pm 7,4$ & $66,5 \pm 2,6$ & $72,1 \pm 7,4$ & $76,9 \pm 5,3$ \\
\hline $\begin{array}{l}W_{\text {máx }} \\
(w)\end{array}$ & $419 \pm 29$ & $420 \pm 42$ & 382 & $413 \pm 36$ & $367 \pm 36$ & $368 \pm 25$ & $392 \pm 35$ & $426 \pm 40$ \\
\hline $\begin{array}{l}W_{\text {max.kg }}{ }^{-1} \\
\left(\text { w. kg }^{-1}\right)\end{array}$ & $6,3 \pm 0,3$ & $5,9 \pm 0,3$ & $5,5 \pm 0,4$ & $6,3 \pm 0,5$ & $5,7 \pm 0,6$ & $5,3 \pm 0,3$ & 6,0 & $6,4 \pm 0,6$ \\
\hline WLL $_{1}$ & $\mathrm{NI}$ & $\mathrm{NI}$ & $\mathrm{NI}$ & $\mathrm{NI}$ & $276 \pm 17$ & $215 \pm 24$ & $286 \pm 32$ & $272 \pm 40$ \\
\hline $\mathrm{WLL}_{2}$ & $\mathrm{NI}$ & $271 \pm 29$ & $\mathrm{NI}$ & $339 \pm 31$ & $318 \pm 14$ & $295 \pm 25$ & $340 \pm 38$ & $360 \pm 29$ \\
\hline
\end{tabular}

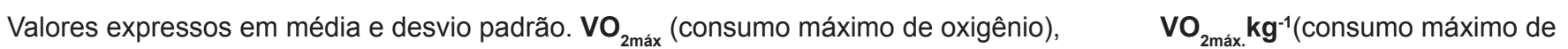
oxigênio relativo), $\mathbf{W}_{\text {máx }}$ (potência aeróbia máxima), $\mathbf{W}_{\text {máx. }} \mathbf{k g}^{-1}$ (potência aeróbia máxima relativa), $\mathbf{W L L}_{1}$ (potência no primeiro limiar de lactato), $\mathbf{W L L}_{2}$ (potência no segundo limiar de lactato), $\mathbf{N I}$ (não informado).

os atletas de XCO apresentam a $\mathrm{W}_{\text {máx }}$ e $\mathrm{VO}_{\text {2pico }}$ relativo à massa corporal significativamente mais alta que os ciclistas $(P=0,03)$.

De fato, ao observarmos as variáveis máximas de aptidão aeróbia apresentadas nas tabelas anteriores, especula-se que maiores valores são encontrados em ciclistas profissionais. Talvez estes achados possam ser explicados em parte por diferenças na biomecânica da pedalada, as adaptações orgânicas provenientes do treinamento e no recrutamento neuromuscular que pode ser distinto devido às diferenças de terreno entre as modalidades. Além disso, os valores altos encontrados nos mountain bikers no estudo de Lee et al. ${ }^{(10)}$ eram esperados, pois alguns participantes foram classificados como um dos melhores do mundo no XCO.

\section{Variáveis Sub-máximas de Aptidão Aeróbia}

A base do conhecimento atual no estudo do comportamento de variáveis fisiológicas em testes progressivos apresenta pontos ou zonas de transição, demarcadores de passagens entre domínios funcionais. Frequentemente os limiares de transições metabólicas (LTM) são referenciados como indicadores de capacidade aeróbia sendo que existe uma grande controvérsia sobre os diferentes métodos de identificação.

Wilber et al.(16) apresentaram que os mountain bikers atingem valores de potência no primeiro limiar de lactato $\left(L L_{1}\right)$ em valores inferiores se comparado aos ciclistas $(271 \pm 29$ vs $321 \pm 17 \mathrm{~W} ; 3,8 \pm 0,3$ vs 4,4 $\pm 0,3$ W.kg-1; $P<0.05)$. Diferente de Lee et al. ${ }^{(10)}$ que utilizaram o modelo Dmáx modificado para identificação do segundo limiar de lactato $\left(L_{2}\right)$ e destacam valores mais expressivos para o grupo de mountain bikers apenas na potência relativa, se comparado aos ciclistas (5,2 $\pm 0,6$ vs 4,7 $\pm 0,3$ W. $\left.\mathrm{kg}^{-1} ; P<0.05\right)$. Apesar destes resultados, de uma forma geral, ciclistas profissionais especialistas em etapas de contra-relógio, atingem valores absolutos bastante elevados de potência no limiar anaeróbio (LA) e "onset of blood lactate accumulation" (OBLA) $(357 \pm 41$ e $409 \pm 46 \mathrm{~W})$ do que atletas de XCO (276 \pm 17 e $318 \pm 14 \mathrm{~W}$ ), respectivamente ${ }^{(2,14)}$. Ao serem corrigidos pela massa corporal os contra-relogistas apresentam valores de OBLA, semelhantes aos 
especialistas em montanhas $(5,7 \pm 0,2$ e $5,7 \pm 0,5$ W. $\left.\mathrm{kg}^{-1}\right)$, sendo também mais destacados que o grupo de mountain bikers $\left(4,9 \pm 0,4 \mathrm{~W} \cdot \mathrm{kg}^{-1}\right)^{(2,14)}$.

As diferenças dos resultados são difíceis de serem explicados e interpretados. Verifica-se que ciclistas profissionais apresentam os valores de potência nos LTM maiores que os mountain bikers. Isto se deve talvez pelo grande volume de treino $\left(\sim 35.000 \mathrm{Km} . \mathrm{ano}^{-1}\right)$, tipo de treinamento e participação em competições, além da sempre presente determinação genotípica(17). Além disso, a eficiência muscular em intensidades severas de ciclistas de alto rendimento parece estar relacionada positivamente com a distribuição de fibras musculares do tipo I na musculatura do vasto lateral|(18). Elevadas proporções de fibras musculares do tipo I, são associadas com o menor consumo de oxigênio para determinadas intensidades, conseqüentemente aumenta a participação do metabolismo aeróbio e eleva a potência produzida, refletindo em maior eficiência muscular ${ }^{(19)}$.

Assim, o conflito relatado na literatura sobre as características fisiológicas de ciclistas e mountain bikers, se expande além das diferenças metodológicas encontradas nos estudos, sendo que talvez estejam presentes no nível competitivo e de treinamento dos diferentes atletas investigados ${ }^{(20)}$. Apesar das variáveis fisiológicas sub-máximas e máximas aqui apresentadas serem superiores para os ciclistas profissionais que disputam as grandes voltas do ciclismo (Tour de France, Giro d'Itália e Vuelta a Espana). Especula-se que, os mountain bikers que apresentam um bom desempenho em competições internacionais de XCO, também se destacam em etapas montanhosas nas grandes voltas do ciclismo, principalmente quando as variáveis fisiológicas são normalizadas pela massa corporal.

\section{Variáveis de Aptidão Anaeróbia}

A capacidade anaeróbia é fundamental para o desempenho em modalidades onde é requerida a manutenção prolongada de grande potência para o fornecimento de energia. A habilidade em gerar picos de potência durante parte do evento (curta duração), tem um papel importante no desempenho do atleta, como por exemplo, nas largadas, subidas, fugas, sprints e principalmente, nas chegadas de uma competição. Nestas condições específicas do esporte, acaba sendo necessária a participação de esforços anaeróbios ${ }^{(4)}$.

Freqüentemente, pesquisadores, técnicos e atletas de alto rendimento estão interessados na identificação de variáveis provenientes de esforços máximos, que sejam fidedignas com o desempenho anaeróbio. No entanto, sobre o conhecimento atual, não existe consenso sobre o método padrão aceito como referencial, havendo pouca concordância sobre o que estes testes realmente avaliam ${ }^{(21)}$. Neste sentido, na tentativa de apresentar evidências de validade, é utilizado o máximo déficit acumulado de oxigênio (MAOD), que representa a diferença entre o equivalente de oxigênio da carga executada e o oxigênio consumido durante o esforço. Esse teste tem uso para estimativa da capacidade anaeróbia a partir da extrapolação linear entre a intensidade de trabalho sub-máxima e $\mathrm{O} \mathrm{VO}_{2}{ }^{(22)}$.

Weber e Schneider(22) examinaram a reprodutibilidade do MAOD medido em $110 \%$ e $120 \%$ do $\mathrm{VO}_{2}$ de pico, em homens e mulheres ciclistas. Seus resultados apontam que não houve diferenças significativas na determinação do MAOD entre os sexos e entre as intensidades, sugerindo aplicação do teste sobre condições padronizadas. Até o presente momento, apenas Dal Monte e Faina ${ }^{(23)}$ utilizaram este método em atletas de XCO sendo encontrado valores de 53,2 $\pm 8,5 \mathrm{mlO}_{2} \cdot \mathrm{kg}^{-1}$. Recentemente, Costa e DeOliveira(24) verificaram as correlações entre variáveis anaeróbias através do Teste de Wingate (TW) com o desempenho de mountain bikers brasileiros não encontrando associações significativas.

Os estudos mais elegantes direcionados em revelar o perfil de ciclistas de estrada profissionais apresentam que as variáveis aeróbias são de extrema importância para o desempenho nas 
competições $^{(9,25,26)}$. No entanto, os mesmos autores simplesmente não apresentam a relevância da participação anaeróbia bem como as avaliações. A participação do componente anaeróbio em ciclistas profissionais que competem no velódromo é bem reconhecida já que as provas são curtas e os atletas imprimem alta potência na bicicleta. Em artigo de revisão, Craig e Norton(27) destacaram a utilização de dinamômetros portáteis durante competições de ciclismo no velódromo. Fica evidente que os altos valores atingidos de potência produzida durante os segundos iniciais dos eventos dependem da participação do metabolismo anaeróbio.

O TW tem sido exaustivamente utilizado para a aproximação da potência e capacidade anaeróbia de ciclistas, com determinação do pico de potência, potência média e índice de fadiga. Tanaka et al. ${ }^{(28)}$ descreveram através do TW, as características anaeróbias de ciclistas norte-americanos. Apesar de ter utilizado uma carga referente a $9,5 \%$ da massa corporal, os autores encontraram valores de 13,9 W. $\mathrm{kg}^{-1} ; 13,6$ W. $\mathrm{kg}^{-1} ; 12,8 \mathrm{~W} . \mathrm{kg}^{-1}$ para 3 categorias diferentes. Baron ${ }^{(4)}$ utilizou um protocolo com duração de 10 segundos para avaliação do componente anaeróbio em diferentes cadências no cicloergômetro isocinético. O autor reportou valores em média de 14,9 W. $\mathrm{kg}^{-1}$, no entanto, estes achados dificilmente podem ser comparados com os existentes devido às diferenças metodológicas.

Heller e Novotny ${ }^{(29)}$ avaliaram a participação anaeróbia de competidores de mountain bike da equipe nacional do Casaquistão. No TW, os autores encontraram valores médios de pico de potência de 14,7 W. $\mathrm{kg}^{-1} \mathrm{e}$ lactato de pico de 11,3 mmol.L-1. Estes achados são semelhantes aos encontrados por Baron(4) quando relativos à massa corporal, no entanto, o protocolo usado pelo autor foi com duração e cargas diferentes. Em adição, o estudo de Heller e Novotny ${ }^{(29)}$ apresenta valores maiores que os valores encontrados por Tanaka et al. ${ }^{(28)}$ isto se justifica já

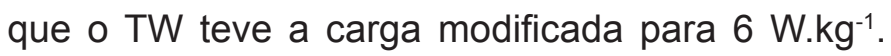
Fica clara a dificuldade de análise desses resultados visto que os diferentes estudos apresentam diferentes metodologias.

\section{Demanda Fisiológica nas Competições de Ciclismo de Estrada e XCO}

Nas grandes voltas do ciclismo os atletas percorrem cerca de $4339 \pm 685 \mathrm{Km}$ durante três semanas consecutivas. A intensidade média de esforço nas competições varia de acordo com as características das etapas. De uma forma geral, o evento é realizado próximo ao primeiro limiar ventilatório (LV1) $)^{(2,9,30)}$. Durante o Tour de France em $1997,70 \%$, 23\% e $7 \%$ do tempo total de dias consecutivos de competição, foram realizadas no domínio fisiológico moderado (até $70 \%$ do $\mathrm{VO}_{2 \text { máx }}$ ), intenso (entre $70 \%-90 \%$ do $\mathrm{VO}_{2 \text { máx }}$ ) e severo (acima de $90 \%$ do $\mathrm{VO}_{2 \text { máx }}$ ), respectivamente, caracterizando a predominância aeróbia do evento ${ }^{(30)}$. A demanda imposta ao organismo durante as competições de ciclismo também está relacionada como tipo de prova que será disputada. Percebe-se que as provas de contra-relógio apresentam a intensidade mais elevada se comparada a provas mais longas. Padilla et al. ${ }^{(31)}$ determinaram a intensidade de esforço durante as provas de contra-relógio através da resposta da FC no Tour de France. Os resultados indicam altos valores entre $88-90 \%$ da $\mathrm{FC}_{\text {máx }}$ no prólogo, sendo que estes valores percentuais diminuem ligeiramente no contra-relógio conforme aumenta-se a distância e a inclinação do percurso $\left(49 \mathrm{~km}=79-84 \%\right.$ da $\left.\mathrm{FC}_{\text {máx }}\right)$.

Em estudo realizado no MTB, Impellizzeri et al. ${ }^{(14)}$ descreveram o perfil da intensidade das competições de XCO através de cardiofrequencímetros. Os autores encontram que ao final das provas, os atletas atingiram a intensidade média correspondente a $90 \pm 3 \% \mathrm{FC}_{\text {máx }}$ É importante ressaltar que foram investigados diferentes tipos de circuito e que apresentaram intensidades semelhantes. Apesar de serem modalidades distintas, estes achados estão de acordo com o perfil das provas de contrarelógio identificadas por Padilla et al. ${ }^{(32)}$. No entanto, 
quando o critério selecionado para caracterizar a sobrecarga de uma competição considera a duração do esforço, podemos afirmar que as provas de XCO apresentam a intensidade mais elevada, pois a duração é de aproximadamente de 2 horas, enquanto que o contra-relógio, geralmente é próximo de 70 minutos $^{(14)}$.

Diversos estudos caracterizam a intensidade de esforço durante as competições com dados calculados a partir do comportamento da FC durante o exercício(14,31,32). No entanto, este método indireto está sujeito a críticas e limitações; a FC pode ser significativamente influenciada pelo "desvio cardiovascular" causado pela desidratação e hipertermia ${ }^{(14)}$. Atualmente, alguns estudos utilizaram dinamômetros portáteis acoplados às bicicletas para registrar a resposta da potência durante o exercício ${ }^{(15,33)}$.

A resposta da potência e FC durante o treinamento e/ou competições também auxilia na determinação no tempo de esforço despendido individualmente em cada domínio fisiológico pré-estabelecidos. Stapelfeldt et al. ${ }^{(15)}$ utilizaram o modelo de zonas de intensidade de esforço, através da análise da resposta da potência, durante as competições de XCO. Neste estudo, os autores classificaram quatro zonas de esforço: abaixo de $L L_{1}$, entre $L L_{1}$ e $L L_{2}$, entre $L L_{2}$ e $W_{\text {máx }}$, e acima da $W_{\text {máx }}$. Impellizzeri et al. ${ }^{14)}$ também utilizaram um modelo semelhante, no entanto através da resposta da FC, sendo classificado em diferentes zonas: leve, moderado e pesado. Assim, os estudos apresentam métodos de identificações de LTM distintos e avaliaram os domínios fisiológicos através da resposta da potência e FC, respectivamente. Neste sentido, Vogt et al. ${ }^{(33)}$ compararam o modelo de zonas de intensidade de esforço, a partir da utilização de monitores de FC e dinamômetros portáteis, durante uma competição de seis dias no ciclismo. Os resultados indicam que a FC subestima o tempo nos domínios abaixo de $L L_{1}$ e acima de $L L_{2}$, sendo que superestima o tempo entre $L L_{1}$ e $L L_{2}$. Assim, devido às grandes oscilações de terreno no XCO, deve-se ter cautela ao estimar os valores de potência e os percentuais de tempo despendido em cada domínio fisiológico, através da FC.

\section{CONCLUSÃO}

Até o presente momento, alguns estudos foram realizados para analisar as respostas fisiológicas durante as competições de ciclismo e XCO. Nesses casos, as pesquisas foram realizadas com atletas profissionais sendo que seus resultados, muitas vezes não podem ser extrapolados para amadores. Para a incorporação de propostas de atletas do exterior no treinamento de atletas brasileiros, é necessário cautela, visto que a pouca informação nacional implicará em utilização talvez equivocada. Dessa forma, entendemos que a interpretação das variáveis morfofisiológicas e competições apresentadas apresentam-se como referências para o treinamento em ciclistas e mountain bikers. De uma forma geral, as características morfológicas dos ciclistas profissionais especialistas em etapas de montanha são semelhantes aos mountain bikers que competem no XCO. Apesar dos estudos aqui apresentados mostrarem informações consistentes sobre as diferenças morfológicas e fisiológicas entre ciclistas especialistas em diferentes terrenos e mountain bikers, acredita-se que as adaptações crônicas provenientes do treinamento de atletas em competições de XCO são semelhantes aos ciclistas especialistas em subidas.

\section{REFERÊNCIAS BIBLIOGRÁFICAS}

1. Union Cyclist International (UCI). The hub [online]. Disponível em: URL: http://www.uci.ch [Acessado em 01 Jun 2008].

2. Mujika I, Padilla S. Physiological and performance characteristics of male professional road cyclists. Sports Med. 2001; 31:479-487.

3. Pfeiffer RP, Kronish RL. Off-road cycling injuries: an overview. Sports Med. 1995; 19 (5): 311-25. 
4. Baron R. Aerobic and anaerobic power characteristics of off-road cyclists. Med Sci Sports Exerc. 2001; 33:1387-93.

5. Swain DP. The influence of body mass in endurance bicycling. Med Sci Sports Exerc.1994; 26: 58-63.

6. Berry JM, Koves TR, Benedetto JJ. The influence of speed, grade and mass during simulated off-road bicycling. Appl Ergon. 2000; 31:531-536.

7. Padilla S, Mujika I, Cuesta G, Goiriena JJ. Level ground and uphill cycling ability in professional road cycling. Med Sci Sports Exerc.1999; 31: 878-885.

8. Costa VP. Variáveis fisiológicas determinantes de performance em mountain bikers. Dissertação de Mestrado em Ciência do Movimento Humano. Centro de Educação Física, Fisioterapia e Desportos. Universidade do Estado de Santa Catarina: Florianópolis, 2006.

9. Lucia A, Hoyos J, Chicharro J. Physiology of professional road cycling. Sports Med. 2001; 31 (7): 325-37.

10. Lee H, Martin DT, Anson JM, Grundy D, Hahn AG. Physiological characteristics of successful mountain bikers and professional road cyclists. J Sports Sci. 2002; 20: 1001-8.

11. Impellizzeri FM, Marcora SM. The physiology of mountain biking. Sports Med. 2007; 37:59-71.

12. Impellizzeri FM, Rampinini E, Sassi A, Mognoni $P$, Marcora S. Physiological correlates to off-road cycling performance. J Sports Sci. 2005a; 23: 41-7.

13. Impellizzeri FM, Marcora SM, Rampinini E, Mognoni $P$, Sassi A. Correlations between physiological variables and performance in high level cross country off road cyclists. Br J Sports Med. 2005b; 39: 747-51.

14. Impellizzeri F, Sassi A, Rodriguez-Alonso M, Mognoni $\mathrm{P}$, Marcora S. Exercise intensity during off-road cycling competitions. Med Sci Sports Exerc. 2002; 34:1808-1813.

15. Stapelfeldt B, Scwirtz A, Schumacher YO, Hillebrecht M. Workload demands in mountain bike racing. Int J Sports Med. 2004;18: 294-300.

16. Wilber RL, Zawadzi KM, Kearney JT, Shannon MP, Disalvo D. Physiological profiles of elite off-road and road cyclists. Med Sci Sports Exerc. 1997; 29: 1090-4.

17. Lucia A, Earnest C, Arribas, C. The Tour de France: a physiological review. Scand J Med Sci Sports. 2003; 13:275-83.
18. Horowitz JF, Sidossis LS, Coyle EF. High efficiency of type I muscular fibers improve performance. Int J Sports Med. 1994; 15:152 - 7 .

19. Coyle EF, Sidossis LS, Horowitz JF. Cycling efficiency is related to the percentage of type I muscle fibers. Med Sci Sports Exerc. 1992; 24:782 - 8.

20. Costa VP, Carminatti LJ, Nakamura FY, De-Oliveira. Morph-Physiological similarities between road cyclists and mountain bikers. Ital J Sports Sci. 2008, 1:11-16.

21. Paton CD, Hopkins WG. Tests of cycling performance. Sports Med. 2001; 31(7): 489-96.

22. Weber CL, Schneider DA. Reliability of MAOD measured at $110 \%$ and $120 \%$ of peak oxygen uptake for cycling. Med Sci Sports Exerc. 2001, 33: 1056-1059.

23. Dal Monte A, Faina M. Valutazione Delli Atleta. pg. 631-645. Editora UTET, Torino, 1999.

24. Costa VP, De-Oliveira. Physiological variables to predict performance in cross-country mountain bike races. J Exerc Physiol. 2008, 11(6):14-24.

25. Padilla S, Mujika I, Cuesta G, Polo JM, Chatard JC. Validity of a velodrome test for competitive road cyclists. Eur J Appl Physiol. 1996; 73: 446-51.

26. Lucia A, Pardo J, Durántez A, Hoyos J, Chicharro JL. Physiological differences between professional and elite road cyclists. Int J Sports Med. 1998; 19:342-348.

27. Craig NP, Norton KI. Characteristics of track cycling. Sports Med. 2001; 31: 457-468.

28. Tanaka H, Basset-JR DR, Swensen TC, Sampedro RM.. Aerobic and anaerobic power characteristics of competitive cycling in the United States Cycling Federation. Int J Sports Med. 1993; 14: 334-338.

29. Heller J, Novotný J. Aerobic and anaerobic capacity in elite mountain bikers. Ac Univ Carol Kin. 1997; 33: 6168.

30. Lucia A, Hoyos J, Carvajal A, Chicharro JL. Heart rate response to professional road cycling: The Tour de France. Int J Sports Med. 1999; 20:167-172.

31. Padilla S, Mujika I, Orbananos J, Angulo F. Exercise intensity during competition time trials in professional road cycling. Med Sci Sports Exerc. 2000; 32: 850-856. 
32. Padilla S, Mujika I, Orbananos J, Santisteban J. Exercise intensity and load during mass-start stage races in professional road cycling. Med Sci Sports Exerc. 2001; 33: $796-802$.

33. Vogt S, Heinrich L, Schumacher YO, Blum A, Roecker $\mathrm{K}$, Dickhuth $\mathrm{HH}$, Schmid A. Power output during stage raging in professional road cycling. Med Sci Sports Exerc. 2006; 38: 147-151.
Endereço para correspondência:

Vitor Pereira Costa

Centro de Educação Superior da Região Sul Universidade do Estado de Santa Catarina Avenida Colombo Machado Salles, s/n - Progresso Laguna - SC - Brasil

CEP 88790-000

e-mail: costavp2@yahoo.com.br 